\title{
Imam et droit pénal : de quelques infractions liées à l'exercice des fonctions cultuelles
}

\section{Vincente Fortier}

\section{(2) OpenEdition}

12 Journals

Édition électronique

URL : http://journals.openedition.org/rdr/434

DOI : $10.4000 /$ rdr.434

ISSN : 2534-7462

Éditeur

Presses universitaires de Strasbourg

\section{Édition imprimée}

Date de publication : 14 novembre 2019

Pagination : 119-134

ISBN : 979-10-344-0054-6

ISSN : 2493-8637

\section{Référence électronique}

Vincente Fortier, «Imam et droit pénal : de quelques infractions liées à l'exercice des fonctions cultuelles », Revue du droit des religions [En ligne], 8 | 2019, mis en ligne le 25 novembre 2019, consulté le 19 novembre 2020. URL : http://journals.openedition.org/rdr/434 ; DOI : https://doi.org/10.4000/rdr. 434

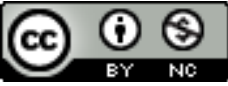

La revue du droit des religions est mise à disposition selon les termes de la Creative Commons Attribution - Pas d'Utilisation Commerciale 4.0 International - CC BY-NC 4.0. 


\section{IMAMET DROIT PÉNAL:DEQUELQUES INFRACTIONSLIÉESS̀ L'EXERCICEDES FONCTIONSCULTUELLES}

\section{Vincente FORTIER}

Université de Strasbourg / CNRS, Droit, Religion, Entreprise et Société (DRES)

\section{RÉSUMÉ}

Un ministre du culte est un citoyen comme un autre et il doit, à ce titre, respecter la loi et répondre de tous les actes répréhensibles qu'il pourrait commettre. À cet égard, la religion est sans incidence sur la responsabilité pénale (ou civile) qui pèse sur le ministre du culte. Plus précisément, celui-ci ne peut pas tirer argument du caractère religieux du mobile l'ayant poussé à commettre une infraction pour échapper à sa responsabilité. Dans cette contribution, seule la responsabilité pénale du ministre du culte directement liée à l'exercice de ses fonctions sera évoquée en distinguant deux infractions principales: la révélation d'une information à caractère secret et la célébration du mariage religieux avant le mariage civil.

\section{Abstract}

A minister of religion is a citizen like any other and as such must comply with law. He must therefore be held accountable for any reprehensible acts he may commit. In this respect, religion does not affect the criminal (or civil) liability of the minister of religion. More specifically, the latter cannot argue that the motive for committing an offence is religious in nature in order to avoid liability. We refer in this paper only to the criminal liability of the minister of religion directly related to the performance of his duties, by highlighting two main offences: the disclosure of secret information and the celebration of religious marriage before civil marriage. 
$\mathrm{U}$ n ministre du culte est un citoyen comme un autre et il doit, à ce titre, respecter la loi et répondre, le cas échéant, de tous les actes répréhensibles qu'il pourrait commettre.

À cet égard, la religion, quels que soient, du reste, les modes d'accession au statut ou à la fonction de ministre du culte, est sans incidence sur la responsabilité pénale (ou civile) qui pèse sur le ministre du culte. Plus précisément, celui-ci ne peut pas tirer argument du caractère religieux du mobile l'ayant poussé à commettre une infraction pour échapper à sa responsabilité. En effet, et en principe, le mobile est indifférent en droit pénal quant à la qualification de l'infraction. Tout au plus, pourrait-il permettre une individualisation de la peine encourue ${ }^{1}$. La chambre criminelle de la Cour de cassation l'a rappelé le 3 septembre $1996^{2}$ à propos d'un imam qui avait procédé à une séance de désenvoûtement d'une jeune fille et qui expliquait que les souffrances n'étaient pas infligées à la victime mais au démon qui était logé en elle. Les juges répondent que «le mobile qui avait pu l'animer, compte tenu de ses convictions religieuses, ne pouvait ôter à ses actes leur caractère d'actes de torture et de barbarie»: les pieds de la victime avaient été flagellés pendant plus de cinq heures, on lui avait fait ingérer une très grande quantité d'eau salée, sa gorge avait été serrée vigoureusement, une serviette enfoncée dans sa bouche, et sa tête plongée dans une bassine d'eau plusieurs fois. De tels traitements avaient entraîné le décès de la jeune fille.

Cette première précision tenant à l'absence d'efficacité du mobile «religieux» étant apportée, sans doute faut-il également s'entendre ici sur le vocabulaire utilisé. En effet, peut-on qualifier l'imam de ministre du culte? Un arrêt de la cour d'appel de Toulouse rendu le 4 juillet $2002^{3}$, qui statuait

1. «Le mobile relève du for intérieur, il est la raison qui pousse l'agent à commettre une infraction. À la différence de l'intention, dol général, qui consiste dans une volonté abstraite, le mobile varie suivant les auteurs: la jalousie, la haine, la colère, le dépit, la cupidité, la plaisanterie, etc. Dans une perspective policière ou criminologique, les mobiles sont importants car, comme l'écrivait Gide, ils constituent "l'anse par laquelle on tient le criminel". Néanmoins, en droit pénal, la loi étant par nature générale et abstraite, les mobiles sont en principe indifférents. Ils ne jouent aucun rôle dans l'opération de qualification, ni comme élément constitutif, ni comme fait justificatif. Ainsi, peut-on commettre un meurtre par jalousie, par cupidité ou par compassion, peu importe: on est meurtrier parce que l'on a eu l'intention de tuer. De même, on peut commettre un vol pour lutter contre le capitalisme ou pour payer ses dettes, peu importe: dès lors qu'il y a soustraction frauduleuse de la chose d'autrui, il y a infraction punissable. En principe, sauf texte contraire, les mobiles "légitimes" ou "honorables" n'exonèrent pas l'auteur» (X. PIN, Droit pénal général, Paris, Dalloz, $8^{e}$ éd. 2017, $\mathrm{n}^{\circ}$ 189).

2. Cass. crim., 3 sept. 1996, $\mathrm{n}^{\circ}$ 96-82.633.

3. CA Toulouse, 4 juill. 2002, $\mathrm{n}^{\circ} 259$ : Jurisdata $\mathrm{n}^{\circ} 2002-204867$. 
sur une demande d'annulation d'exclusion de deux membres d'une association cultuelle musulmane, prend le parti d'une réponse en creux: «Si l'imam n'a pas la qualité de ministre du culte, la confiance et l'autorité morale que lui attribuent les fidèles donnent aux injures à son encontre un caractère de particulière gravité». En réalité, et du point de vue légal, il n'existe aucune définition de la notion de ministre du culte, même si la loi vise expressément celui-ci pour certaines infractions. Cette absence de définition n'est pas surprenante en régime de séparation, l'État laissant aux institutions et groupements religieux le soin de s'organiser et de déterminer les contours et les modes d'accès aux fonctions de ministres du culte, en vertu du principe d'autodétermination ${ }^{4}$. Dès lors, la notion renvoie à des statuts bien différents d'une religion à une autre. Toutefois, et de manière générale, le titre de ministre du culte est conféré à une personne qui occupe, la plupart du temps après une période probatoire, une fonction primordiale dans la direction et le déroulement d'un culte. On rappellera que selon la jurisprudence administrative $^{5}$, le culte comprend notamment les cérémonies, les rites, les formations (séminaires, etc.). L'existence d'une hiérarchie ecclésiastique et de différentes appellations n'a qu'un intérêt purement interne à la religion en question.

Sur le plan juridique, on retiendra que le ministre du culte est la personne chargée de l'exercice d'un culte, lequel peut prendre différentes formes et composantes. Il peut donc y avoir plusieurs catégories de ministres du culte au sein d'une même et seule religion.

Ces précisions étant apportées, seule la responsabilité pénale du ministre du culte directement liée à l'exercice de ses fonctions sera ici évoquée, en distinguant deux infractions principales: la révélation d'une information à caractère secret (1) et la célébration du mariage religieux avant le mariage civil (2).

4. Dans un arrêt du 19 octobre 1999, rendu à propos de l'obligation au secret à laquelle sont tenus les ministres du culte, et particulièrement dans cette affaire les ministres du culte des Témoins de Jéhovah, la cour d'appel de Montpellier précise: «Attendu qu'il n'existe aucune définition légale du ministre du culte dans la loi du 9 décembre 1905 relative à la séparation des Églises et de l'État et que le pouvoir nominatif de définir cette notion est reconnu aux églises; qu'en l'espèce, le juge ne peut contester la nomination par le Consistoire national des témoins de Jéhovah de ses ministres du culte, qui sont par ailleurs qualifiés de "bergers spirituels" » (CA Montpellier, 19 oct. 1999: Jurisdata n 1999-113307).

5. CE, avis ass., 24 oct. 1997, n 187122: Lebon: «Il résulte des dispositions des articles 18 et 19 de la loi du 9 décembre 1905 [...] que les associations revendiquant le statut d'association cultuelle doivent avoir exclusivement pour objet l'exercice d'un culte, c'est-à-dire, au sens de ces dispositions, la célébration de cérémonies organisées en vue de l'accomplissement, par des personnes réunies par une même croyance religieuse, de certains rites ou de certaines pratiques» : RFDA 1998, p. 61-69, note G. GonZalez; P. Rolland, "Qu'est-ce qu'un culte aux yeux de la République?», ASSR, n 129, 2005, p. 51-63: http://journals. openedition.org/assr/1109 [consulté le 15 mai 2019]. 


\section{LA RÉVÉLATION D'UNE INFORMATION À CARACTÈRE SECRET}

L'obligation au secret à laquelle sont astreints les ministres du culte trouve aujourd'hui son fondement dans l'article 226-13 du Code pénal qui dispose: «La révélation d'une information à caractère secret par une personne qui en est dépositaire soit par état ou par profession, soit en raison d'une fonction ou d'une mission temporaire, est punie d'un an d'emprisonnement et de 15000 euros d'amende. »

Ce texte, entré en vigueur le $1^{\text {er }}$ mars 1994, rénove la présentation des personnes tenues au secret ${ }^{6}$. En effet, le texte ancien, soit l'article $378 \mathrm{du}$ Code pénal de 1810, désignait nommément certains professionnels astreints au secret (médecins, chirurgiens et autres officiers de santé, ainsi que les pharmaciens, les sages-femmes) y ajoutant «toutes autres personnes dépositaires par état ou profession ou par fonctions temporaires ou permanentes des secrets qu'on leur confie». La doctrine et la jurisprudence, suppléant l'imprécision du texte, s'accordèrent pour soumettre au secret professionnel les «confidents nécessaires», c'est-à-dire ceux qui exercent une profession ou qui ont un état les destinant à recevoir des secrets qu'il est nécessaire de leur confier. Parmi ceux-ci, trouvons-nous les ministres du culte. Comme le souligne Thierry Massis:

«Contrairement à l'article 378 de l'ancien Code pénal, la loi n'énumère plus certaines catégories de professions; elle vise la profession, l'état, la fonction qui conduisent une personne à être dépositaire d'une information à caractère secret. Cette distinction n'est pas une simple subtilité de juriste. La comparaison entre les deux textes révèle que ce n'est plus seulement la confidence qui est protégée, mais toute information secrète découverte dans l'exercice d'une activité professionnelle, d'une fonction quelconque, voire d'une mission ${ }^{7}$. »

L'obligation au secret qui s'impose aux ministres du culte, et qui corrélativement les protège, est une création prétorienne dont tout à la fois l'étendue, la portée et les assujettis ont été déterminés au fil des espèces.

6. L'article 226-13 ne fait plus allusion à des professions en particulier, mais définit l'auteur de l'infraction comme la personne qui révèle une information à caractère secret si elle « en est dépositaire soit par état ou par profession soit en raison d'une fonction ou d'une mission temporaire». V. sur ce point, Ch. CHOMIENNE et Ch. GuÉRY, «Secret, révélation, abstention ou les limites de la liberté de conscience du professionnel dans le nouveau Code pénal », ALD 1995, comm. 85.

7. Th. MAssis, «La transparence et le secret. Champ social, débat de conscience», Études, 2001/6, p 751-761: https://www.cairn.info/revue-etudes-2001-6-page-751.htm [consulté le 15 mai 2019]. 
Il serait, toutefois, inexact de croire que la prise en compte du secret des ministres du culte ne date que de $1810^{8}$. L'obligation au secret de ces personnes est ancienne et l'article 378 n'a en réalité innové qu'en fixant une peine équivalente à tous les auteurs d'une révélation couverte par le secret professionnel.

Les ordonnances royales comme les canons de l'Église interdisaient de révéler le secret de la confession. Le sceau de la confession devait être inviolable et les confidences reçues au tribunal de la pénitence ensevelies «dans un silence éternel ». La jurisprudence des parlements protégeait alors la religion catholique dans l'un de ses rites essentiels, la confession, mais limitait l'obligation de secret aux seuls faits appris en confession. Cette jurisprudence, fort intéressante ${ }^{9}$, a beaucoup évolué au fil du temps quant aux dépositaires du secret religieux, à la nature de celui-ci et à l'étendue de l'obligation au secret.

Les dépositaires de secret religieux sont bien évidemment les prêtres et les religieux catholiques, mais également les religieux non ordonnés, les diacres et aujourd'hui les laïcs amenés à participer à l'administration et à la distribution des sacrements. La qualité de dépositaire est aussi dévolue aux

8. L'obligation au secret qui pèse sur les ministres du culte trouve sa source dans la pratique de la confession privée de l'Église chrétienne. Aux $\mathrm{II}^{\mathrm{e}}$ et $\mathrm{III}^{\mathrm{e}}$ siècles, il ne pouvait être question de secret de la confession puisque celle-ci était publique; cependant, pour les péchés secrets, fut instaurée la pratique du prêtre pénitencier afin de pallier les dangers de la pénitence publique. Il s'agissait alors d'un tête-à-tête entre le pénitent et le prêtre permettant à ce dernier de juger de l'attitude du pécheur, d'apprécier le degré de gravité de sa faute et de lui imposer une pénitence. Le prêtre était alors tenu au secret, en vertu d'un devoir moral affirmé avec force par les théologiens du IV siècle sans que, pour autant, l'Église n'intervînt en prévoyant des sanctions contre ceux qui violeraient ce devoir de silence. Les premières prescriptions qui imposent expressément aux confesseurs le silence apparaissent dans plusieurs canons syriens et arméniens punissant de déposition le prêtre qui aura révélé le contenu d'une confession. Il faut attendre le pontificat de Grégoire VII pour que de sévères mesures soient prescrites à l'encontre des prêtres violant le secret de la confession: ceux-ci étaient déposés et condamnés à faire toute leur vie des pèlerinages. En 1215, le canon 21 du IV Concile du Latran édicta: "Celui qui aura découvert le péché qu'il aura connu par la voie de la confession sera, par notre ordonnance, non seulement déposé de la dignité sacerdotale, mais encore enfermé dans un monastère d'étroite observance, pour y faire pénitence le reste de ses jours». Le principe de la confession secrète est adopté et l'obligation au silence imposée aux confesseurs. $\mathrm{Au} \mathrm{XVI}^{\mathrm{e}}$ siècle, le Concile de Trente confirma le principe du secret absolu (M. RoBinE, « Le secret professionnel du ministre du culte», D. 1982, chron., p. 221; L. Ribaut, L'évolution du secret professionnel du ministre du culte, mém. DEA, Montpellier, 1994; B. du Puy-Montbrun, «La détermination du secret chez les ministres du culte, son histoire et son droit», Annuaire Droit et Religions, 2013-2014, p. 15-36.

9. Pour l'étude de cette évolution, V. notre article, «Le secret des ministres du culte», in J.-P. Royer et B. Durand, Secret et justice, le secret entre éthique et technique, Lille, Publ. de l'Espace juridique, 2000. 
pasteurs de l'Église protestante, aux titulaires d'une délégation pastorale, aux conseillers presbytéraux et aux femmes de pasteurs, de même qu'aux rabbins et aux représentants des autres cultes. Ainsi, et concernant la religion musulmane, l'imam doit-il respecter les dispositions sur le secret professionnel.

Quant à la nature du secret, la seule invocation de la qualité de ministre du culte ne permet pas de refuser de révéler une information. En effet, l'information doit présenter un caractère secret au sens de l'article 226-13 du Code pénal. Ce sont, en réalité, les circonstances de la confidence faite au ministre du culte qui déterminent ou non l'obligation de se taire pesant sur lui.

L'étendue de l'obligation au secret concerne, au premier chef, ce que le ministre du culte a appris en confession. Ceci n'intéresse que les catholiques et le respect rigoureux de la confession n'a jamais été contesté. Toutefois, les prêtres, à l'instar des ministres du culte d'autres religions, sont amenés à recevoir des confidences qui ne sont pas couvertes par le secret de la confession.

Une distinction est ici faite entre les faits connus ou confiés aux ministres du culte dans l'exercice de leurs fonctions et ceux qu'ils auraient pu connaître en dehors ou abstraction faite de leur ministère. Dès 1810, la jurisprudence se prononçait en faveur du secret pour des aveux faits à un prêtre hors confession ${ }^{10}$. Plus tard, une jurisprudence constante a toujours admis que sont protégés contre toute divulgation les faits confiés à un ministre du culte dans l'exercice ou à l'occasion de ses fonctions. C'est en 1891 que la Cour de cassation ${ }^{11}$ va prononcer l'arrêt de principe dans le domaine, en étendant explicitement la matière couverte par le secret pastoral et en qualifiant l'obligation au secret d'absolue et d'ordre public. La question se posait très

10. La limite tenant aux aveux faits en confession fut repoussée en 1810 par la cour française de Turin (Turin, 28 févr. 1810: S. 1811, 2, p. 63), décision rendue alors que le Code pénal n'était pas encore en vigueur. Un dénommé Bonino, accusé de vol, avait été condamné par le tribunal correctionnel. Celui-ci avait puisé la preuve du délit dans les aveux faits par l'accusé à la partie lésée, par l'ordre de son confesseur, qui l'avait obligé à demander pardon, sous peine d'être privé de l'absolution. Bonino fit appel, soutenant que ces aveux ayant été faits par suite de sa confession étaient essentiellement secrets et que leur révélation ne pouvait être considérée aux yeux des tribunaux comme constituant la preuve du délit. La cour de Turin fit droit à sa demande. Bonino fut libéré, la cour ayant considéré que les aveux faits hors de la confession, mais déterminés par le secret dû à cet acte ne pouvaient servir de preuve en justice. De la même façon, ceux qui ont reçu de tels aveux ne peuvent être contraints de témoigner. Ainsi en décida la Cour de cassation, le 30 novembre 1810 (crim., 30 nov. 1810: S. 1811, 1, p. 49) qui, visant les articles du Concordat, considéra que «si, en l'espèce, la révélation n'avait pas eu lieu réellement dans un acte religieux et sacramentel de confession, elle n'avait été déterminée que par le secret dû à cet acte».

11. Cass. crim., 4 déc. 1891: D. 1892, 1 139, obs. Sallantin, concl. Baudoin. 
clairement de savoir si le prêtre était tenu de déposer sur des faits, révélés en dehors de la confession, mais qui étaient confiés à lui à raison de son caractère sacerdotal. La Cour de cassation considéra que: «Les ministres des cultes légalement reconnus sont tenus de garder le secret sur les révélations qui ont pu leur être faites à raison de leurs fonctions; pour les prêtres catholiques, il n'y a pas lieu de distinguer s'ils ont eu connaissance des faits par la voie de la confession ou en dehors de ce sacrement. Cette circonstance ne saurait changer la nature du secret dont ils sont dépositaires si les faits leur ont été confiés dans l'exercice exclusif de leur ministère sacerdotal et à raison de ce ministère. Cette obligation est absolue et d'ordre public.» Les termes de l'obligation au secret des ecclésiastiques étaient ainsi définitivement arrêtés: l'étendue et la portée de cette obligation ne varieront guère au fil du temps.

Le secret ne concerne pas seulement les prêtres catholiques pour des faits par eux connus en confession ou dans l'exercice de leur ministère. Il est également dû par les ministres des autres cultes qui ne connaissent pas la confession.

Lorsque les faits sont révélés à des ministres du culte pour des raisons étrangères à leur mission religieuse, par exemple l'amitié, la parenté ou la compétence dans un domaine extra-religieux, ces faits ne relèvent pas du secret religieux et donc ne sont pas couverts par le secret. Dans différentes affaires, les juges ont pu rappeler que l'obligation de garder les faits secrets ne peut être imposée aux ministres du culte pour les faits dont ils ont eu connaissance non comme ministres du culte, mais comme hommes, amis ou parents ${ }^{12}$.

Le secret religieux auquel sont astreints les ministres du culte est une notion complexe. Et cela d'autant plus que le secret professionnel est évoqué par la loi pénale dans plusieurs textes prévoyant tantôt l'obligation de se taire (c'est l'article 226-13 du Code pénal), tantôt l'obligation de parler. Celle-ci concerne le ministre du culte comme du reste tout citoyen et découle des articles 434-1 ${ }^{13}$,

12. V. par ex. CA Toulouse, 14 mars 1928: S. 1928, 2, p. 130: «si lesdites confidences ont été faites à un homme particulièrement apte, par sa dignité, par sa profonde connaissance de la nature humaine à les recevoir et à ordonner le conseil qu'on lui demandait, elles ne lui étaient pas faites en tant que confident nécessaire, en raison directe de sa qualité de religieux et des pouvoirs sacerdotaux dont il était investi.»

13. «Le fait, pour quiconque ayant connaissance d'un crime dont il est encore possible de prévenir ou de limiter les effets, ou dont les auteurs sont susceptibles de commettre de nouveaux crimes qui pourraient être empêchés, de ne pas en informer les autorités judiciaires ou administratives est puni de trois ans d'emprisonnement et de 45000 euros d'amende. [...]

Sont également exceptées des dispositions du premier alinéa les personnes astreintes au secret dans les conditions prévues par l'article 226-13.» 
$434-3^{14}$ et $434-11^{15}$ du Code pénal. D'autres textes peuvent également emporter nécessité pratique de divulguer au moins en partie un secret confié, par exemple l'obligation de porter secours à personne en péril ${ }^{16}$.

Dès lors, si la révélation d'un secret religieux est envisageable, sa mise en œuvre demeure complexe. Ainsi, l'article 434-1 du Code pénal sanctionne la non-dénonciation de crime. Il prévoit cependant expressément que cette obligation ne s'applique pas aux personnes astreintes au secret professionnel dans les conditions prévues par l'article 226-13 du Code pénal. L'absence de dénonciation par une personne tenue au secret professionnel d'un crime dont elle aurait eu connaissance ne saurait alors être sanctionnée pénalement et la possibilité de signalement à l'autorité judiciaire de certains faits, prévue par l'article 226-14 du Code pénal, est une simple faculté, laissée à la discrétion du débiteur du secret, et non une obligation.

Quant à l'article 434-3 du Code pénal, il prévoit également en son second alinéa que cette obligation (de dénoncer) ne s'applique pas aux personnes

14. «Le fait, pour quiconque ayant connaissance de privations, de mauvais traitements ou d'agressions ou atteintes sexuelles infligés à un mineur ou à une personne qui n'est pas en mesure de se protéger en raison de son âge, d'une maladie, d'une infirmité, d'une déficience physique ou psychique ou d'un état de grossesse, de ne pas en informer les autorités judiciaires ou administratives ou de continuer à ne pas informer ces autorités tant que ces infractions n'ont pas cessé est puni de trois ans d'emprisonnement et de 45000 euros d'amende.

Lorsque le défaut d'information concerne une infraction mentionnée au premier alinéa commise sur un mineur de quinze ans, les peines sont portées à cinq ans d'emprisonnement et 75000 euros d'amende.

Sauf lorsque la loi en dispose autrement, sont exceptées des dispositions qui précèdent les personnes astreintes au secret dans les conditions prévues par l'article 226-13.»

15. «Le fait, pour quiconque connaissant la preuve de l'innocence d'une personne détenue provisoirement ou jugée pour crime ou délit, de s'abstenir volontairement d'en apporter aussitôt le témoignage aux autorités judiciaires ou administratives est puni de trois ans d'emprisonnement et de 45000 euros d'amende.

Toutefois, est exempt de peine celui qui apportera son témoignage tardivement, mais spontanément. [...]

Sont également exceptées des dispositions du premier alinéa les personnes astreintes au secret dans les conditions prévues par l'article 226-13.»

16. C. pén., art. 223-6: «Quiconque pouvant empêcher par son action immédiate, sans risque pour lui ou pour les tiers, soit un crime, soit un délit contre l'intégrité corporelle de la personne s'abstient volontairement de le faire est puni de cinq ans d'emprisonnement et de 75000 euros d'amende.

Sera puni des mêmes peines quiconque s'abstient volontairement de porter à une personne en péril l'assistance que, sans risque pour lui ou pour les tiers, il pouvait lui prêter soit par son action personnelle, soit en provoquant un secours.

Les peines sont portées à sept ans d'emprisonnement et 100000 euros d'amende lorsque le crime ou le délit contre l'intégrité corporelle de la personne mentionnée au premier alinéa est commis sur un mineur de quinze ans ou lorsque la personne en péril mentionnée au deuxième alinéa est un mineur de quinze ans.» 
astreintes au secret professionnel, tout en ajoutant «sauf lorsque la loi en dispose autrement $»$. Cette incise semble concerner essentiellement les personnes participant aux missions du service de l'aide sociale à l'enfance et les assistants de service social qui sont, pourtant, tenus au secret professionnel (CASF, art. L. 221-6) ${ }^{17}$.

Nous constatons, ici encore, que les personnes tenues au secret professionnel sont autorisées à signaler l'existence des faits énoncés par l'article 434-3 du Code pénal, mais elles n'y sont pas obligées. Ces personnes (ministres du culte compris) disposent d'une option de conscience.

La question centrale, en réalité, sera de savoir si l'information revêt ou non un caractère secret, ce qui emporte la nécessité pour les juridictions répressives d'examiner avec attention les circonstances dans lesquelles l'information a été reçue. L'affaire du cardinal Barbarin récemment jugée par le tribunal correctionnel de Lyon ${ }^{18}$ est un exemple topique de cette situation. En effet, Philippe Barbarin avait eu connaissance des faits d'agressions sexuelles par l'une des victimes (si l'on prend comme point de départ la lettre écrite par la victime en juillet 2014) qui, comme le relève le tribunal, avait manifestement l'intention de les divulguer. Il ne s'agissait point d'une information apprise dans le secret du confessionnal, ni même d'une confidence en lien avec l'exercice des fonctions ecclésiastiques du cardinal. La démarche de la victime n'était nullement confidentielle dans la mesure où elle s'était ouverte des faits à d'autres personnes. Dès lors, cette information n'était pas couverte par le secret professionnel. En conséquence, Philippe Barbarin ne disposait pas de l'option de conscience: il était tenu d'informer la justice des faits dont il avait eu connaissance.

\section{LA CÉLÉBRATION DU MARIAGE RELIGIEUX AVANT LE MARIAGE CIVIL}

Aux termes de l'article 433-21 du Code pénal, «Tout ministre d'un culte qui procédera, de manière habituelle, aux cérémonies religieuses de mariage sans que ne lui ait été justifié l'acte de mariage préalablement reçu par les officiers de l'état civil sera puni de six mois d'emprisonnement et de 7500 euros d'amende».

17. Circulaire relative au secret professionnel des ministres du culte et aux perquisitions et saisies dans les lieux de culte, CRIM 2004-10 E1/11-08-2004, NOR: JUSD0430163C.

18. TGI Lyon, 7 mars 2019: Dalloz actualités, 18 mars 2019, obs. S. Fucinile. 
Bien que l'article 433-21 du Code pénal ait «adouci» les sanctions encourues par le ministre du culte en cas de commission de l'infraction en instaurant une infraction d'habitude, par rapport à l'article 199 du Code pénal de 1810, le maintien de cette antériorité du mariage civil sur le mariage religieux et, plus précisément le caractère obligatoire du mariage civil si les époux souhaitent se marier religieusement, sont discutés par certains ${ }^{19}$. Tel est notamment le cas de Michel Legrain ${ }^{20}$. Émile Poulat s'interrogeait également sur cette situation:

«Si l'État ne peut imposer le mariage civil à des concubins notoires, peut-il encore l'imposer à des croyants convaincus qui ont leurs raisons de s'en tenir au mariage religieux? Et si ceux-ci invoquaient la protection internationale européenne ou simplement constitutionnelle de leur liberté de conscience et de culte? À ce recours juridique, ils semblent jusqu'ici préférer les solutions de contrebande ou de clandestinité comme l'Église en a prévues pour les situations extraordinaires ${ }^{21}$.»

De fait, dans son ouvrage consacré aux imams ${ }^{22}$, Solenne Jouanneau dédie des pages extrêmement intéressantes à «la redéfinition du mariage musulman en France» et au rôle joué par les imams. Elle indique également que le principe juridique de primauté du mariage civil sur le mariage religieux est rejeté par certains fidèles:

«Les imams bénévoles rencontrés mobilisent généralement deux registres de justification pour expliquer ce refus. Le premier est d'ordre idéologique: certains musulmans par souci d'orthopraxie ou par conviction politique refuseraient la primauté (tant symbolique que temporelle) du mariage civil laïque sur le mariage religieux, considérant en quelque sorte que "ce qui n'est pas l'islam ne peut venir légitimer ce qui perpétue ce même islam"; le second est d'ordre plus prosaique et aurait

19. Lors des discussions relatives au nouveau Code pénal, la disparition des incriminations prévues aux articles 199 et 200 anciens avait été envisagée, en raison notamment de leur peu d'intérêt. En Allemagne, l'obligation d'antériorité du mariage civil sur le mariage religieux a été supprimée dans le cadre de la loi du 19 février 2007 portant réforme de l'état civil, entrée en vigueur le $1^{\text {er }}$ janvier 2009 (F. CURTIT, «Allemagne: suppression de l'obligation d'antériorité du mariage civil sur le mariage religieux», Société, droit et religion, 2011/1, p. 117-124: https://www.cairn.info/revue-societe-droit-et-religion-20111-page-117.htm [consulté le 15 mai 2019]).

20. M. Legrain, «Mariage civil et mariage religieux, se marier à l'église sans passer par la mairie?», RDC 50/1, 2000, p.163-169.

21. La Croix, 26-27 août 1990, cité par M. LEgRAin dans son article précité.

22. S. JouAnneau, Les imams en France, une autorité religieuse sous contrôle, Marseille, Agone, 2013. 
trait à la volonté de certaines familles de faire coïncider mariage civil et autonomie matérielle effective des jeunes couples ${ }^{23}$.»

Solenne Jouanneau ajoute toutefois une troisième cause de rejet dont elle explique qu'elle est dissimulée: «légitimer ou rendre acceptable [...] ce qui dans les faits relève plus d'une union libre que d'un véritable mariage ». Dès lors, il ne s'agirait pas «d'un acte de contestation ou de négation de la légitimité d'un État laïque à encadrer les unions matrimoniales, mais relèverait d'une stratégie visant à rendre pour soi et pour les autres, socialement respectable ou religieusement légitime une mise en couple que l'on souhaite pouvoir rompre à tout moment sans contrainte excessive ${ }^{24} »$.

La réaction des imams face à la demande des fidèles de célébration d'un mariage religieux sans être passés par la mairie est variable: certains contournant la loi sans s'y opposer frontalement, d'autres refusant d'y souscrire.

Romain Sèze relève également qu'existe en France une demande de mariage religieux musulman émise par de jeunes couples, qui, sur le modèle de leurs pairs chrétiens, souhaitent «passer devant l'imam ${ }^{25}$ ». L'auteur souligne que:

«Dans une société où les pratiques religieuses ne relèvent plus d'une évidence sociale, l'imam symbolise le dernier bastion d'un ordre qui s'évanouit et doit de fait s'afficher. Réside ici toute la valeur du certificat de mariage que délivrent depuis peu quelques imams en France (pratique observée dans plusieurs mosquées). La régulation normative se déploie dans des espaces communs aux différentes autorités religieuses. En ce sens, la sécularisation est bien productrice de religieux ${ }^{26}$.»

Toutes les religions sont concernées par le texte de l'article 433-21 du Code pénal, même si la religion catholique était initialement visée. Et de la même manière que pour le secret religieux, on doit entendre par ministre du culte celui qui, selon le droit interne de la religion concernée, est considéré comme tel.

Les dispositions visent les mariages religieux, et par conséquent une cérémonie qui ne présenterait pas un caractère religieux, ni celui d'un mariage (bénédiction, fiançailles) ne tombe pas sous le coup de l'article 433-21 du Code pénal.

26. Ibid., p. 120. 
Ensuite l'infraction n'est constituée que si le mariage religieux est antérieur au mariage civil: cela implique que le ministre du culte n'a pas exigé des époux la présentation du certificat de mariage, du livret de famille ou d'un extrait d'acte de mariage. Par conséquent, n'est réprimée que l'absence de justification de la célébration civile. La difficulté de la situation tient à la preuve, car les mariages religieux sont souvent clandestins. Peu importe le délai séparant les deux célébrations, ou que le ministre du culte ait dû célébrer un mariage in extremis en raison du danger de mort de l'un des conjoints ${ }^{27}$. Enfin, comme il s'agit d'une infraction d'habitude, il faut que soient établies contre le ministre du culte au moins deux célébrations religieuses irrégulières.

Il faut noter que le contentieux est fort rare dans ce domaine. Cela ne signifie pas qu'il n'y a pas de célébration de mariage religieux ou de pseudo mariage par des imams: les enquêtes de Solenne Jouanneau tout comme celles de Romain Sèze prouvent l'inverse. Peut être cité, avec circonspection, le jugement du tribunal correctionnel de Meaux du 24 février $2011^{28}$ à l'encontre d'un imam ayant prétendument célébré soixante-dix-sept mariages religieux. En réalité, et en appel, devant la cour de Paris le 17 janvier $2013^{29}$, il ne restait plus que neuf mariages ou soi-disant mariages à partir de l'enquête diligentée par le procureur de la République. Et au final, le prévenu a été relaxé au bénéfice du doute! La décision indique:

«Il résulte de plusieurs témoignages que l'épouse n'a pas assisté à la cérémonie conformément à la tradition et que les cinq témoignages ne contiennent aucune précision sur le déroulement de la cérémonie son sens réel et l'implication de l'imam auquel il apparaît que le droit musulman ne confère pas de rôle particulier dans la célébration des mariages religieux. Il demeure donc un doute quant à la nature des cérémonies pratiquées avant le mariage civil ainsi que quant à sa présence même quand bien même ces personnes ont pu en retirer la conviction qu'il s'agissait de cérémonies de mariage les autorisant à se comporter comme époux.»

En l'espèce, l'imam reconnaissait avoir récité des prières de bénédiction sans faire aucune déclaration lors de cérémonies de fiançailles et il soulignait qu'il n'avait pas le pouvoir de donner des droits aux personnes, en précisant

27. F. Messner, P.-H. Prélot, J.-M. Woehrling (dir.), Traité de droit français des religions, Paris, Litec, $2^{\mathrm{e}}$ éd. 2013, $\mathrm{n}^{\mathrm{os}}$ 1537-1539.

28. Inédit.

29. CA Paris, 17 janv. 2013, $n^{\circ} 11 / 08385$ : Jurisdata $n^{\circ} 2013-001818$. 
que la majorité des gens ne savaient pas ce qu'était le mariage en islam et faisaient le plus souvent la confusion entre les cérémonies organisées en France et les pratiques dans les pays où l'islam est religion d'État.

Ces développements sont intéressants car ils se retrouvent dans les ouvrages consultés sur l'imamat. Tous les auteurs font état de ces mariages religieux dont sont saisis les imams. Toutefois, dans leurs interviews, les imams précisent toujours qu'ils n'ont pas le pouvoir d'unir les couples, que le mariage est un contrat, mais qu'en France ils sont appelés à s'occuper des mariages pour en vérifier les bases religieuses et les conditions du contrat d'alliance. Il est nommé par les fidèles le mariage halal.

Quoi qu'il en soit et, du reste, quel que soit l'avis porté sur cette primauté du mariage civil sur le mariage religieux, il convient de ne pas ignorer les dangers potentiels nés de ces situations de mariage seulement religieux et dont les femmes principalement sont victimes. En effet, le mariage religieux ne produit, en France, aucun effet civil; il ne peut donc pas créer de liens juridiques entre les personnes, contrairement à ce que celles-ci pourraient parfois croire.

En janvier 2019, le Conseil théologique des imams du Rhône s'est penché, lors de sa deuxième assemblée générale, sur l'articulation entre droit civil et droit religieux en matière de mariage et de divorce ${ }^{30}$, rappelant à tous les imams du Rhône "qu'ils ne doivent en aucun cas célébrer un mariage religieux si celui-ci n'est précédé d'un mariage civil à la mairie ou devant toute autre institution officielle». Corroborant les enquêtes produites dans les ouvrages, les imams font état de la pression des parents lorsque leurs enfants commencent à se fréquenter. Toutefois, selon les propos rapportés dans le journal La Croix, "les imams qui acceptent sont de plus en plus rares parce qu'ils ont peur de l'amende. Mais les familles trouvent des "imams" ou prédicateurs de passage, d'autant plus facilement qu'ils se font payer pour cela».

L'un des problèmes majeurs à résoudre tient à celui des musulmanes engagées dans un mariage religieux, mais dont le couple n'existe plus. Or celles-ci ne parviennent pas à obtenir de leur mari la déclaration formelle de répudiation qui leur permettrait de refaire leur vie. Cette situation ne serait pas exceptionnelle, rendue encore plus difficile en présence d'enfants que le conjoint refuse de reconnaître. Pour régler ces situations au plan religieux, il est préconisé la création d'une commission composée d'imams pour tenter, en première intention « une réconciliation du couple». Toutefois, «si aucune

30. A.-B. HoffNer, «Les imams du Rhône plaident pour le mariage civil », La Croix, 14 janv. 2019. 
solution n'est envisageable, la commission peut prendre la décision de considérer la femme comme séparée, même si son mari ne veut pas prononcer la formule légale ou coutumière du divorce ${ }^{31} »$.

Par ailleurs, il ne faut pas ignorer les menaces qui pèsent sur les plus jeunes femmes, très souvent mineures, manipulées par des hommes rencontrés sur les réseaux sociaux et mariées par téléphone. On peut bien évidemment se poser la question de savoir si une célébration à distance peut être qualifiée de mariage. Observons que, pour déterminer ce qui constitue une cérémonie du mariage au sens du Code pénal, il faut se reporter aux règles internes des confessions considérées: toute définition légale est prohibée. Rien ne permet $a$ priori d'exclure de la qualification de mariage religieux, un mariage contracté par téléphone (ou par Skype). En effet, "peu importe que le mariage religieux célébré soit religieusement valable ou non, pourvu que l'officiant ait cru qu'il l'était; peu importe que l'officiant n'ait pas en personne participé à la cérémonie religieuse du mariage, le rite particulier choisi ne requérant pas sa présence effective auprès des futurs conjoints ${ }^{32} »$. Or les situations peuvent devenir absolument dramatiques. Ainsi en est-il de cette affaire survenue en 2013 et jugée le 3 septembre 2015 par le tribunal correctionnel de Mulhouse ${ }^{33}$. L'adolescente avait 15 ans et le prévenu principal, âgé de 28 ans au moment des faits, était poursuivi pour «soustraction d'enfant des mains d'une personne chargée de sa garde» et «abus de la faiblesse». La jeune fille, convertie depuis un an en cachette de ses parents chrétiens, "par téléphone et en passant par des réseaux sociaux sur Internet», selon ses propos rapportés par les enquêteurs, souhaitait «se marier» religieusement à un musulman pratiquant. Elle fut mise en relation avec un candidat (le prévenu) habitant à Valence, dans la Drôme. Celui-ci était déjà marié, père de deux enfants et recherchait «une seconde épouse musulmane pour soulager la première épouse dans les tâches ménagères, s'occuper des enfants et le combler sexuellement».

Après avoir correspondu par mails, les deux personnes ont échangé leurs engagements respectifs par téléphone. "Il n'y avait pas d'imam, ce n'était pas un mariage», précisa le prévenu. Le 28 avril, l'adolescente invita son "promis» à la rejoindre en Alsace. Lors de son audition, la jeune fille a indiqué qu'il s'agissait là en fait de son deuxième "mariage», car elle avait déjà été répudiée par un premier «époux».

31. Ibid.

32. F. Messner, P.-H. Prélot, J.-M. Woehrling (dir.), op. cit., $\mathrm{n}^{\circ} 1538$.

33. Le Monde, 15 mai 2013; L'Alsace, 30 sept. 2015. 
L'homme a été relaxé pour les faits d'enlèvement, mais condamné pour abus de faiblesse à deux ans de prison, dont vingt mois avec sursis. Le tribunal lui a interdit tout contact avec la victime. Celle-ci et son père obtiennent respectivement 1500 et $1000 €$ de dommages et intérêts.

Cette affaire est à rapprocher de celle traitée en 2016 par la chambre spéciale des mineurs de la cour d'appel de Rouen ${ }^{34}$ à propos de la radicalisation religieuse d'une jeune fille mineure. Celle-ci s'était aussi secrètement mariée religieusement avec un jeune homme au domicile des parents de ce dernier à Saint-Étienne-du-Rouvray. Le mariage avait été célébré par un imam syrien par téléphone. Elle avait connu le jeune homme quelques mois auparavant, par l'intermédiaire d'une amie commune impliquée dans un réseau islamiste.

Dans les deux affaires relatées qui revêtent une particulière gravité, la disposition du Code pénal sur l'antériorité du mariage civil sur le mariage religieux n'aurait vraisemblablement rien empêché, et par ailleurs, comment poursuivre le ministre du culte? Toutefois, cela engage les imams à la plus extrême prudence et à un devoir de vigilance à l'égard de leurs fidèles. Par ailleurs, le rappel de la loi est toujours le bienvenu. Le mariage civil ne constitue-t-il pas un moyen efficace pour lutter contre les mariages forcés, dans la mesure où l'officier d'état-civil doit s'assurer du consentement libre de chacun des époux ${ }^{35}$ ?

\section{CONCLUSION}

À ces deux infractions relevant du Code pénal susceptibles d'engager la responsabilité pénale des imams comme de tout ministre du culte, nous pourrions ajouter celles prévues spécifiquement par la loi du 9 décembre 1905: d'une part, l'outrage ou la diffamation commis par un ministre du culte (art. 34 al. 1 de la loi de $1905^{36}$ ); d'autre part, la provocation à la résistance aux

34. CA Rouen, 11 oct. $2016, \mathrm{n}^{\circ} 16 / 03439$ : JurisData $\mathrm{n}^{\circ}$ 2016-022986.

35. V. FORTIER, «La règle contre le rite: l'offensive du droit français contre les mariages forcés », in M.-F. BUREAU (dir.), Sexualité et démocratie, perspectives multidisciplinaires francophones, Éd. Revue de Droit de l'Université de Sherbrooke, 2009, p. 125-156.

36. «Tout ministre d'un culte qui, dans les lieux où s'exerce ce culte, aura publiquement par des discours prononcés, des lectures faites, des écrits distribués ou des affiches apposées, outragé ou diffamé un citoyen chargé d'un service public, sera puni d'une amende de 3750 euros et d'un emprisonnement d'un an, ou de l'une de ces deux peines seulement.

La vérité du fait diffamatoire, mais seulement s'il est relatif aux fonctions, pourra être établie devant le tribunal correctionnel dans les formes prévues par l'article 52 de la loi 
lois (art. 35 de la loi de 1905 37 ). Toutefois, le champ de ces infractions est étroit et le contentieux concernant ces deux dispositions est ancien et se situe entre 1906 et 1915.

Enfin, une autre affaire ${ }^{38}$ peut être signalée relative à l'application de l'article 24 alinéa $1^{\text {er }}$ de la loi du 29 juillet 1881 sur la liberté de la presse. Il s'agissait d'un imam (très précisément l'imam de Vénissieux) qui avait accordé une interview à un journaliste. Cet entretien avait été publié et dans cet article il engageait les hommes à battre leur femme infidèle très fort! Il fut poursuivi pour provocation directe à la commission d'une atteinte à l'intégrité physique de la personne sur la base de l'article précité.

En première instance, il fut relaxé. Sur appel du ministère public et de la partie civile (une association), la cour d'appel de Lyon le condamna à 6 mois d'emprisonnement avec sursis et $2000 €$ d'amende. L'imam forma un pourvoi en cassation qui fut rejeté par la chambre criminelle. Le prévenu faisait valoir que, «s'étant borné à rappeler les termes du Coran, il n'avait pas eu l'intention de provoquer à commettre des atteintes à la personne». En réalité, selon l'arrêt, le prévenu ne s'était pas exprimé par référence au Coran et il avait librement développé ses propos sur l'autorisation donnée aux hommes de frapper leur femme sans évoquer sur ce point l'interdit posé par la loi française. Il avait donc intentionnellement provoqué à la commission du délit de violence volontaire sans pouvoir se prévaloir d'aucune cause d'irresponsabilité pénale.

du 29 juillet 1881. Les prescriptions édictées par l'article 65 de la même loi s'appliquent aux délits du présent article et de l'article qui suit. »

37. «Si un discours prononcé ou un écrit affiché ou distribué publiquement dans les lieux où s'exerce le culte, contient une provocation directe à résister à l'exécution des lois ou aux actes légaux de l'autorité publique, ou s'il tend à soulever ou à armer une partie des citoyens contre les autres, le ministre du culte qui s'en sera rendu coupable sera puni d'un emprisonnement de trois mois à deux ans, sans préjudice des peines de la complicité, dans le cas où la provocation aurait été suivie d'une sédition, révolte ou guerre civile.»

38. Cass. crim., 6 févr. 2007, n 05-86.495: Jurisdata n 2007-037948. 